\title{
Distributional Lexical Semantics for Stop Lists
}

\author{
Mr Neil Cooke \\ University of Surrey \\ n.cooke@surrey.ac.uk \\ Dr Lee Gillam \\ University of Surrey \\ I.gillam@surrey.ac.uk
}

\begin{abstract}
In this paper, we consider the use of techniques that lead naturally towards using distributional lexical semantics for the automatic construction of corpora-specific stop word lists. We propose and evaluate a method for calculating stop words based on collocation, frequency information and comparisons of distributions within and across samples. This method is tested against the Enron email corpus and the MuchMore Springer Bilingual Corpus of medical abstracts. We identify some of the data cleansing challenges related to the Enron corpus, and particularly how these necessarily relate to the profile of a corpus. We further consider how we can and should investigate behaviours of subsamples of such a corpus to ascertain whether the lexical semantic techniques employed might be used to identify and classify variations in contextual use of keywords that may help towards content separation in "unclean" collections: the challenge here is the separation of keywords in the same or very similar contexts, that may be conceived as a "pragmatic difference". Such work may also be applicable to initiatives in which the focus is on constructing (clean) corpora from the web, deriving knowledge resources from wikis, and finding key information within other textual social media.
\end{abstract}

Stop words, lexical distributional semantics, information retrieval.

\section{INTRODUCTION}

Distributional lexical semanticists are interested in deriving information about words and the concepts they refer to using, for example, statistical approaches over vector space models. Part of the applicable hypothesis relates to Firth's comment regarding a word and its company. This is extended to considerations of lexical semantics in which words sharing similar company may relate to one another either through some shared super-type, a synonymy relation, or some variation therein. Pustejovsky, Anick and Bergler (1993) explored the combined use of a representation for lexical knowledge, a generative lexicon, and computations of Mutual Information over known syntactic classes. The authors cite Hindle's drink-based example, how it is possible to create a metric for the substitutability of a set of words within certain contexts depending on the extent of shared contexts of those words. For distributional lexical semantics: 'tea' and 'coffee' may share a number of collocations, e.g. drink. The more that are shared, the more likely it becomes that these items are either synonymous or belong to the same class. When investigated more robustly, beyond simple co-occurrence, this becomes a comparison of collocation pattern.

In our work, we are considering whether models of collocation distributions can be used to derive stop word lists from arbitrary text corpora. The principle we use is similar to the work cited above, though we are attempting in part to derive the set of contexts, rather than the words themselves, based on sets of words selected at random from the corpus. Other research into the construction of stop word lists makes use of Zipf's law (Watts, Porter, \& Zhu. 2002, Lo, et.al 2005, Trumbach \& Payne 2007), similarity tests against pre-classified document sets (Wilbur \& Sirotkin 1992; Hiemstra \& de Jong 2001; Sinka \& Corne 2003, Lo, et.al 2005), and specific selections of sets of reference data (Blei, et al. 2003; Fumera, et al. 2006). Such approaches result in a variety of stop word lists, hence a variety of corpus linguistics research being undertaken with variations in repeatability of this research. In addition, it is assumed that such corpora are "clean", largely since they have been manually constructed. The stop lists tend to have a limited relationship with the corpus at hand, and a limited mathematical basis (Hiemstra \& de Jong 2001). Our work draws on analysis of neighbourhoods of collocation patterns (Smadja 1993; Levy \& Bullinaria 2001) and investigates statistically specific (narrow) peaks of these patterns, in combination with other measures used in characterising distributions in corpora (Gillam and Ahmad 2005). We consider, specifically the problem of separation of data based on pragmatic differences: the same word may be used in apparently identical syntactic and semantic ways, but much wider consideration is needed to establish the pragmatic differences. We initially discuss corpus profiling and Zipfian distributions, with consideration for how to identify certain types of problems with distributions within corpora (section 2), then discuss the Enron corpus, and our prior work relating to the problems of information pollution (section 3), and duplication of text "objects", which may skew results of any analysis (section 4). We then discuss stop list research and the approach for calculating stop word lists and Distributional Lexical Semantics and present automatic extraction and distributional similarity examples from the Enron corpus and the Much-more 
corpus to demonstrate our approach (section 5). Finally we discuss the need for sub-sampling, and some considerations for future efforts (section 6).

\section{CORPUS PROFILING AND ZIPFIAN DISTRIBUTIONS}

The distribution of tokens in a text is considered as an important property in information theory. Through manual analysis of James Joyce's Ulysses, George Kingsley Zipf posited a power-law function using the notion that the rank of a token, when ordered by frequency, is inversely proportional to its frequency (Zipf 1949). Researchers have since identified that rank multiplied by relative frequency produces a constant around 0.1 (Li 1992, Yavuz 1974); rank multiplied by frequency produces a constant that is a tenth the number of tokens in the corpus (e.g. for the Brown Corpus, Manning and Schutze 1999, pp26-27).

Corpora, however, do not appear to be so well behaved. Consideration of the British National Corpus (BNC), comprising 100,106,029 word-tokens with 669417 different word-types, demonstrates this: Zipf's law suggests that the constant is around 10 million. The data, on the other hand, do not follow this [Table 1] and, indeed, only between ranks around 500 to 3500 does this approximate easily.

\begin{tabular}{llll}
\hline Rank (r) & Frequency (f) & $\mathbf{f}^{*} \mathbf{r}$ & \% Error \\
\hline 1 & 6187927 & 6187927 & $-38.19 \%$ \\
2 & 2941790 & 5883580 & $-41.23 \%$ \\
3 & 2682878 & 8048634 & $-19.60 \%$ \\
4 & 2560346 & 10241384 & $+2.31 \%$ \\
5 & 2150885 & 10754425 & $+7.43 \%$ \\
$\ldots \ldots$ & & & \\
3001 & 3417 & 10254417 & $+2.44 \%$ \\
3002 & 3414 & 10248828 & $+2.38 \%$ \\
$\ldots \ldots$ & & & \\
4991 & 1746 & 8712540 & $-12.95 \%$ \\
4992 & 1746 & 8714286 & $-12.98 \%$ \\
$\ldots$. & & & \\
65521 & 26 & 1703546 & $-82.98 \%$ \\
65522 & 26 & 1703572 & $-82.98 \%$
\end{tabular}

TABLE 1: Error values - Zipfian expectations

Indeed, the same is true for the Brown Corpus. However, our consideration of Zipf's law is not for the purpose of suggesting corpora are Zipfian, or to suggest that Zipf-Mandelbrot provides a better fit, but to suggest that Zipf's law provides a simple computable benchmark against which characteristics of other corpora may be contrasted: we consider that deviations (error values in contrast to Zipfian expectations) may provide a signature for the distribution of the corpus.

High frequency words behave in certain ways, with the top 100 most frequent variously accounting for a large proportion of the tokens in the corpus - around $40-50 \%$. But it is also interesting to consider that about $53 \%$ of words in the BNC have a frequency of 1 - and since the number of types is rather less than $1 / 10$ of the size of the corpus, Zipf's law breaks for obvious reasons. For words of frequency 1, their relative rank in a frequency list is arbitrary but their proportions are interesting. Consider frequencies from 1 to 10 in 5 specialist text corpora with a predicted derived value derived from Zipf's law that suggests the proportion of words occurring with a given frequency, $f$, is $1 / f(f+1): 50 \%$ for $f=1,12 \%$ for $f=2$, and so on. Values are shown in Table 2 .

\begin{tabular}{|c|c|c|c|c|c|c|}
\hline Frequency & Automotive & Nuclear & Finance & Nanotech & BNC & Predicted \\
\hline Tokens & 350920 & 393993 & 685037 & 1012096 & 100106029 & \\
\hline Types & 14252 & 14937 & 28793 & 26861 & 669417 & \\
\hline 1 & $38.44 \%$ & $40.55 \%$ & $36.76 \%$ & $43.28 \%$ & $52.84 \%$ & $50.00 \%$ \\
\hline 2 & $14.14 \%$ & $15.18 \%$ & $15.20 \%$ & $13.50 \%$ & $12.92 \%$ & $16.70 \%$ \\
\hline 3 & $7.14 \%$ & $6.73 \%$ & $8.58 \%$ & $7.01 \%$ & $6.02 \%$ & $8.30 \%$ \\
\hline 4 & $5.10 \%$ & $4.85 \%$ & $5.78 \%$ & $4.66 \%$ & $3.71 \%$ & $5.00 \%$ \\
\hline 5 & $3.64 \%$ & $3.29 \%$ & $3.99 \%$ & $3.46 \%$ & $2.54 \%$ & $3.30 \%$ \\
\hline 6 & $2.69 \%$ & $3.05 \%$ & $2.96 \%$ & $2.64 \%$ & $1.90 \%$ & $2.40 \%$ \\
\hline 7 & $2.15 \%$ & $2.19 \%$ & $2.30 \%$ & $1.95 \%$ & $1.51 \%$ & $1.80 \%$ \\
\hline 8 & $1.78 \%$ & $1.83 \%$ & $1.82 \%$ & $1.73 \%$ & $1.20 \%$ & $1.40 \%$ \\
\hline 9 & $1.80 \%$ & $1.41 \%$ & $1.54 \%$ & $1.33 \%$ & $0.98 \%$ & $1.10 \%$ \\
\hline 10 & $1.48 \%$ & $1.33 \%$ & $1.45 \%$ & $1.19 \%$ & $0.83 \%$ & $0.90 \%$ \\
\hline Total & $\begin{array}{l}78.36 \% \\
\text { TABLE 2: }\end{array}$ & $\begin{array}{l}80.42 \% \\
\text { ecialist Co }\end{array}$ & $\begin{array}{l}\mathbf{8 0 . 3 8 \%} \\
\text { ora, Error }\end{array}$ & $\begin{array}{l}80.76 \% \\
\text { ues - Zipfial }\end{array}$ & $\begin{array}{l}\mathbf{8 4 . 4 5 \%} \\
\text { \{pectations }\end{array}$ & $90.90 \%$ \\
\hline
\end{tabular}

Specialist corpora, it appears, are less prone to large vocabularies in comparison to the representative general language corpus (BNC) and the Zipfian prediction, which BNC most closely follows. Another consideration here is that these low frequencies may also reflect errors in spelling, and other untidy formulations within the source data. 
On the other hand, it becomes a possibility requiring consideration that increased repetition is an artefact of repeated texts or, potentially, text fragments across numerous documents. We consider some of these issues in relation to the Enron corpus in the following section.

\section{ENRON CORPUS "BURYING THE BAD NEWS"?}

The Enron corpus is one of the largest business email collections available for public use from the Federal Energy Regulatory Commission (FERC). The most widely available version ${ }^{1}$ comprises around 150 email accounts with 3349 folders, totalling some 517,431 files. Unlike most corpora, the Enron corpus was not designed or, originally, selected. It was largely a dump of the contents of the email server and hence presents particular challenges in addition to its size. Much Enron corpus research to date has focused on issues of data cleansing, for example, deduplication, but also on elements of social network analysis, deception analysis and small scale automatic classification efforts. Table 3, published previously in, (Cooke, Gillam \& Kondoz 2007a\&b) provides a brief summary of this research.

\begin{tabular}{|c|c|c|}
\hline Application & $\begin{array}{l}\text { Corpus size } \\
\text { (\# emails) }\end{array}$ & Further Description \\
\hline $\begin{array}{l}\text { Automatic } \\
\text { classification }\end{array}$ & 12,500 & $\begin{array}{l}\text { Determining whether emails are for "Business" or "Personal" uses, University of } \\
\text { Sheffield, UK, } \\
\text { (Jabbari et al 2006) }\end{array}$ \\
\hline $\begin{array}{l}\text { Data cleansing, } \\
\text { Preliminary } \\
\text { analysis }\end{array}$ & 200,399 & $\begin{array}{l}\text { Analysis of email threads and message distribution. } \\
\text { Some folders removed, Carnegie Mellon } \\
\text { (Klimt \& Yang 2004) }\end{array}$ \\
\hline $\begin{array}{l}\text { Annotation; } \\
\text { visualisation }\end{array}$ & 1,700 & $\begin{array}{l}\text { Manual annotation of email categories. } \\
\text { http://bailando.sims.berkeley.edu/enron email.html } \\
\text { University of California, Berkeley }\end{array}$ \\
\hline & 255,636 & $\begin{array}{l}\text { Visualisation and clustering. Use of database structure separating bodies, } \\
\text { headers and other elements. University of California, Berkeley } \\
\text { http://bailando.sims.berkeley.edu/enron email.html }\end{array}$ \\
\hline $\begin{array}{l}\text { Automatic } \\
\text { classification }\end{array}$ & 20,581 & $\begin{array}{l}\text { Automatic approach to building email folders Massachusetts Amherst } \\
\text { (Bekkerman, McCallum \& Huang 2004) }\end{array}$ \\
\hline $\begin{array}{l}\text { Data } \\
\text { de-duplication }\end{array}$ & 250,485 & $\begin{array}{l}\text { MD5 Hashes on body text to identify duplicates, resulting in } 250,485 \text { emails. } \\
\text { Massachusetts, Amherst } \\
\text { http://ciir.cs.umass.edu/ } / \text { corrada/enron/ } \\
\text { TABLE 3: Related Enron corpus research }\end{array}$ \\
\hline
\end{tabular}

We initially consider two characterisations of the Enron corpus to explore how certain expectations may or may not hold: by frequency and by weirdness. In this analysis we contrast two versions of the corpus, the Enron (raw) $500 \mathrm{k}$ email Enron corpus ${ }^{1}$ and Enron-CleanUCB a de-duplicated 200k email Enron corpus provided by Klimt \& Yang (2004). We know in advance that the raw Enron email corpus provides at least two challenges, (i) vast amounts of duplication which distorts frequency analysis; (ii) presence of email headers, which distorts relationship analysis. Enron (clean) has, reportedly, removed email headers and duplicates.

The first 100 most frequent "words" in Enron-Raw account for $45 \%$ of the corpus; at frequencies up to $10,77 \%$ of the vocabulary is accounted for with only $27 \%$ at a frequency of 1 . We might summarise that such a corpus is demonstrating specialist tendencies, but this should be considered inconclusive. Contrasting the top 10 most frequent words between Enron-Raw and Enron-Clean (Table 4), it would appear that the initial list would have been dominated by elements of email headers (e.g. enron, com, $\mathrm{x}, \mathrm{cn}$ ); stop words have been impacted both by removal of duplicates and subject lines.

\begin{tabular}{llll}
\hline $\begin{array}{l}\text { Enron-Raw } \\
\text { Word }\end{array}$ & Frequency & $\begin{array}{l}\text { Enron-CleanUCB } \\
\text { Word }\end{array}$ & Frequency \\
\hline enron & $7,555,888$ & the & $2,806,643$ \\
com & $6,881,814$ & to & $2,025,907$ \\
the & $5,684,275$ & r & $1,896,214$ \\
to & $5,072,137$ & and & $1,286,641$ \\
$x$ & $3,654,791$ & of & $1,180,840$ \\
and & $2,593,183$ & a & $1,077,861$ \\
of & $2,391,399$ & in & 862,776 \\
cn & $2,332,235$ & enron & 766,304
\end{tabular}

It would appear, then, that the clean version would now be a much more appropriate corpus for further analysis. Consider, however, a tag cloud of tokens discovered with highest weirdness (highly frequent and unusual in comparison to the BNC) for both corpora (Figure 1 and Figure 2): 


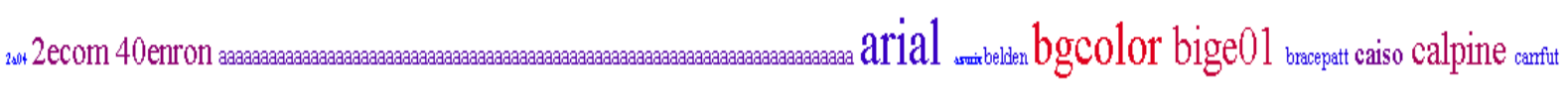
cellpadding cellspacing colspan com cpuc dabhol dasovich dbcaps 97 data delainey $d y n e g y$ earthink ect edu ees

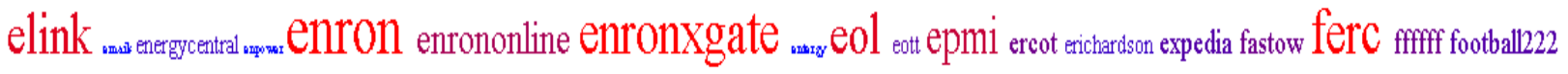

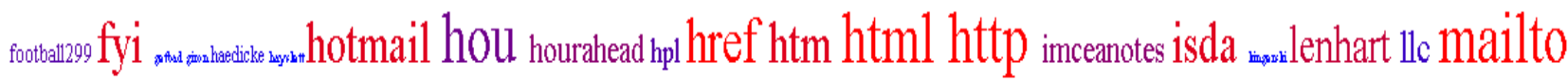

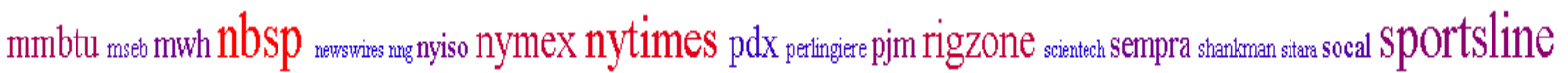
steffes $\mathrm{td} 2$ todaysheadlines transwestem travelocity ${ }_{\mathrm{m}}$ unSubscribe url valign venturewire verdana website ${ }_{\text {westdesk wac }}$ WWW Xls

FIGURE 1: Raw Enron Tag cloud

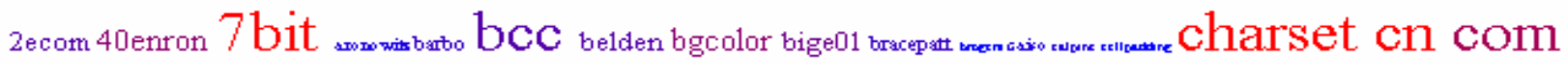
cpuc dasovich dec2000 delainey donoho dynegy ect elink enron enrononline

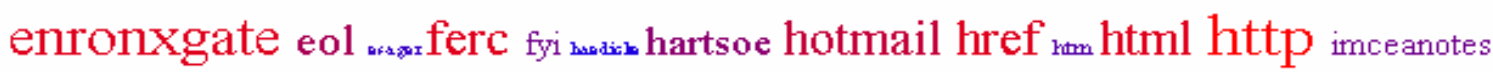
inbox isda jan2002 jamnold javamail jlavora jsteffe jun2001 june2001 kearey kingerski kmann

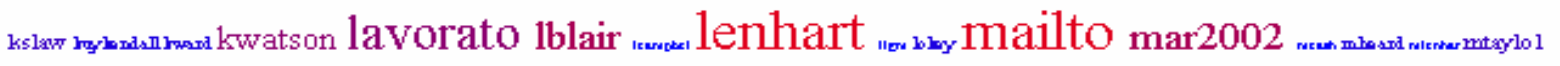

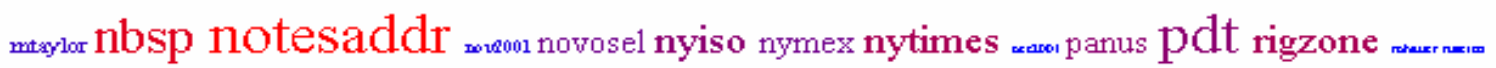
schoolcraft amensempra shankman skean sportsline sscous sshackl steffes stokley tana td2 tholt tjones tycholiz ongowebsite WWW

FIGURE 2: Clean Enron Tag cloud

Enron-Raw contains numerous indications of the corpus being polluted with a variety of items; Enron-CleanUCB shares a decent proportion of these polluting items, and further work is required on this corpus to be able to deal effectively with such content. Generally, this requires techniques to separate, and to document the separation, of specific kinds of data; ideally, to produce a system of multidimensional markup that leaves the "original" corpus intact. It may be possible, then, to propose a set of mechanisms, involving initial benchmarking against Zipf's law, to identify corpora that are unusually distributed, as appears to be the case in Enron.

\section{FINDING ENRON'S CONFIDENTIAL INFORMATION}

Our reason for conducting the analysis above stems from our search for context. We are undertaking research into email filtering for information security and intellectual property protection, and need to ensure that we can reduce false positives and do not produce any false negatives. In the course of our work (Cooke, Gillam \& Kondoz 2007a\&b) with the Enron corpus and in contrast to work normally undertaken with collocation patterns (Smadja 1993) we considered collocation patterns over window sizes around 150 words to identify textual "zones". Typically, collocation distances of 5 are considered; as part of this work we have been attempting to establish whether such collocation distances are justified for all kinds of analysis, and outcomes indicative that it may not be appropriate always to make such an a priori selection of distance. This extended zone collocation analysis of "confidential" has shown that the dominant register is that of a "confidentiality" banner. An example is shown figure 3:

DISCLAIMER: This e-mail message is intended only for the named recipient(s) above and may contain information that is privileged, confidential and/or exempt from disclosure under applicable law. If you have received this message in error, or are not the named recipient(s), please immediately notify the sender and delete this e-mail message.

FIGURE 3: Example "confidentiality" banner

Such banners, often around 80 words in size, with a significant number of variants reaching 130 words, provide a textual "zone" that will act as an analytical distraction for a number of candidate keywords including law, privileged, exempt and disclosure. Banners emerging from different organisations are variously constructed, and this is before we begin to consider variations comprising banners in multiple languages, company address information, anti-virus scanning statements and so on. These will be variously scattered throughout such collections.

Table 5 presents the most frequent collocations with "confidential". Table 6 presents dominant collocations after removal of stop words: using the 2000 most frequent words of the British National Corpus (BNC) as the stop list, [a typical approach to stop lists (Blei, et al. 2003; Fumera, et al. 2006)]. 


\begin{tabular}{llllllllllll}
\hline Collocate & Total & L5 & L4 & L3 & L2 & L1 & R1 & R2 & R3 & R4 & R5 \\
\hline and & 30584 & 623 & 300 & 7512 & 1961 & 3310 & 15262 & 768 & 230 & 372 & 246 \\
may & 15264 & 307 & 1144 & 161 & 10290 & 5 & 74 & 2815 & 249 & 190 & 29 \\
contain & 11004 & 1613 & 205 & 428 & 7 & 8630 & 0 & 0 & 38 & 83 & 0 \\
the & 13886 & 459 & 1485 & 681 & 160 & 473 & 151 & 551 & 966 & 667 & 8293 \\
for & 10129 & 493 & 377 & 149 & 88 & 116 & 105 & 296 & 629 & 7228 & 648 \\
privileged & 19390 & 68 & 13 & 1375 & 4780 & 1647 & 71 & 6599 & 2593 & 1398 & 846 \\
material & 6367 & 2 & 0 & 30 & 0 & 0 & 21 & 22 & 5122 & 1153 & 17 \\
relevant & 4863 & 4856 & 7 & 0 & 0 & 0 & 0 & 0 & 0 & 0 & 0 \\
information & 11143 & 688 & 1013 & 704 & 271 & 123 & 5379 & 338 & 1111 & 715 & 801 \\
affiliate & 5051 & 185 & 4855 & 7 & 0 & 0 & 0 & 0 & 0 & 0 & 4
\end{tabular}

TABLE 5: "Confidential" collocations BNC

\begin{tabular}{llllllllllll}
\hline Word & Total & L5 & L4 & L3 & L2 & L1 & R1 & R2 & R3 & R4 & R5 \\
\hline contain & 11004 & 1613 & 205 & 428 & 7 & 8630 & 0 & 0 & 38 & 83 & 0 \\
privileged & $\mathbf{1 9 3 9 0}$ & $\mathbf{6 8}$ & $\mathbf{1 3}$ & $\mathbf{1 3 7 5}$ & $\mathbf{4 7 8 0}$ & $\mathbf{1 6 4 7}$ & $\mathbf{7 1}$ & $\mathbf{6 5 9 9}$ & $\mathbf{2 5 9 3}$ & $\mathbf{1 3 9 8}$ & $\mathbf{8 4 6}$ \\
affiliate & 5051 & 185 & 4855 & 7 & 0 & 0 & 0 & 0 & 0 & 0 & 4 \\
legally & 4724 & 3 & 1117 & 342 & 71 & 0 & 70 & 139 & 2475 & 499 & 8 \\
intended & 3990 & 69 & 17 & 4 & 0 & 0 & 10 & 2535 & 516 & 570 & 269 \\
exempt & 2480 & 0 & 0 & 0 & 0 & 0 & 0 & 430 & 218 & 1832 & 0 \\
proprietary & 3097 & 147 & 107 & 1726 & 70 & 7 & 649 & 258 & 129 & 3 & 1 \\
unauthorized & 1415 & 0 & 0 & 0 & 0 & 0 & 0 & 0 & 8 & 0 & 1407 \\
solely & 1399 & 0 & 0 & 1 & 0 & 0 & 0 & 9 & 1275 & 98 & 16 \\
email & 2864 & 63 & 510 & 932 & 506 & 1 & 5 & 4 & 21 & 5 & 817
\end{tabular}

TABLE 6: "Confidential" collocations BNC top 2000 removed

These collocates demonstrate strong distributional associations within this neighbourhood, and within the wider neighbourhood of our analysis, demonstrating the prevalence of confidentiality banners. In order to find any confidential material within such a corpus, it would first be necessary to subtract the confidentiality banners.

\section{STOP! WORDS!}

Previous analysis has demonstrated that there are several kinds of polluting information within the Enron corpus. Since these are artefacts of the corpus, we reflect on how we may remove stop words using a completely arbitrary stop list that has no relationship to the texts, nor any mathematical basis (Hiemstra \& de Jong 2001). These distributions of pollution will cause difficulties to the uninitiated. Similarly, inappropriate stop lists can have severe effects on vector based semantic representations (Bullinaria \& Levy 2006).

Typical approaches to constructing stop lists are to use Zipf's law (Watts, Porter, \& Zhu. 2002, Lo et al 2005, Trumbach \& Payne 2007) or comparison to other pre-classified document sets and performing a "similarity test" (Wilbur \& Sirotkin 1992; Hiemstra \& de Jong 2001; Sinka \& Corne 2003, Lo et al, 2005), both of which have little foundation on the text under analysis.

Collocation analysis reveals many interesting shapes and patterns. We claim that collocations whose patterns look like flat lines across the collocation positions, up to five words to the left (L5 to L1) and up to five words to the right (R1 to R5) of the example nucleating word "energy" as shown in figure 4 , have little relationship to the nucleate and hence have stop word characteristics. Typically, analysis methods use frequency as a means for stop list construction and standard deviation for ranking analysis, but ranking methods based on standard deviation are strongly biased by frequency. We are interested in words that have a strong distance relationship represented by a statistically significant narrow peak ${ }^{2}$ and we claim that these have entropy and represent local context.

Our formulae below exploit both unusual frequency (cohesion - strength of the peak) and the narrowness of the peak (local context - strength of the association) to automatically rank the collocates and hence derive the stop list. We consider single peak patterns or "High Q" shapes, e.g. mission; flat lines, e.g. as, and locking exclusions e.g. jones, vice, would, as collocations with "energy" (Figure 4).

The High $Q$ terms are combined with relative frequency using formula 1,2 and 3 . Relative Frequency is based on the whole corpus, we analysis the shapes that emerge from consideration of such patterns.

$Q_{-}$peak $=\frac{\sum_{i=1}^{n} F i^{2}}{F^{2}}$

\footnotetext{
$F=$ total Frequency of the collocate

$\mathrm{Fi}=$ Frequency of the collocate at word distance $I=n$

$n=$ number of collocation positions
}

${ }^{2} \mathrm{~A}$ high resonant $\mathrm{Q}$ in $\mathrm{RF}$ filter terminology 


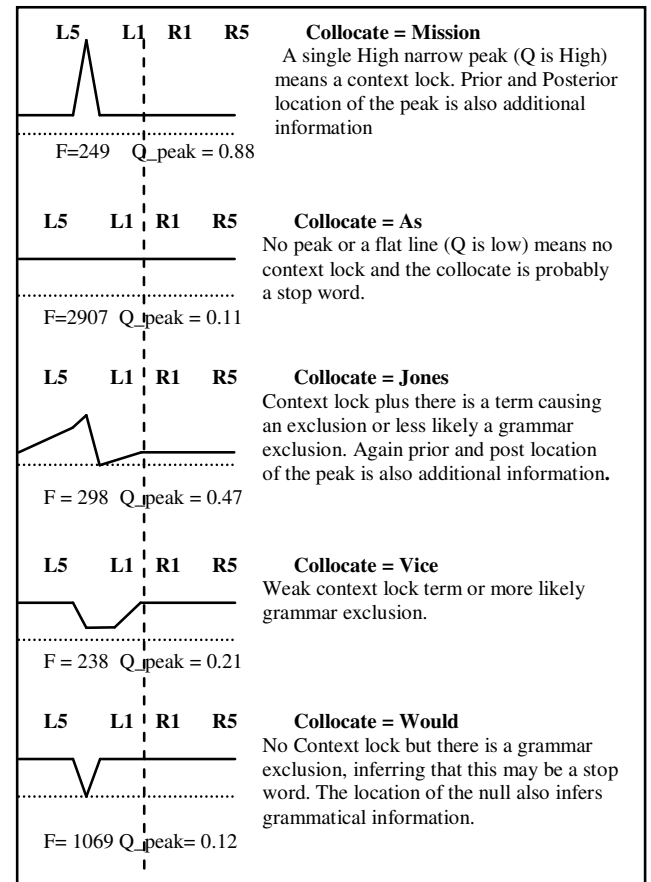

FIGURE 4: Dasovich ${ }^{3}$ "energy" collocation pattern examples

$$
W=\frac{F_{S L} N_{G L}}{\left(F_{G L}+1\right) N_{S L}}
$$

$F S L=$ word frequency in target corpus

$N G L=$ reference corpus size (tokens)

$N S L=$ target corpus size (tokens)

$F G L=$ words frequency in reference corpus

Weirdness is exponential and combined as a cubed root to reduce its aggressiveness for unseen words.

$$
Q w=Q_{-} \text {peak } \times W^{1 / 3}
$$

To generate the stop list we arbitrarily chose 1.2 as the cut off point for Qw, setting the value higher increases the Stop list aggression.

When reading, we use both knowledge (through relatedness) and observational experience (through similarity) to disambiguate words. Knowledge disambiguates through conceptual relationships: this approach requires both a validated knowledge base and heuristics (Budanitsky \& Hirst, 2006). Observational experience uses Semantic Similarity and is based on human perceptions of frequently co-occurring words: if we observe that cat is frequently near animal then if platypus is also frequently near animal we may infer similarity between similarity between platypus and cat (Levy \& Bullinaria, 2001). This is a lexical semantic similarity approach, and we can use such relationships to distinguish and associate. If two nucleates share strong High $Q$ words then we can infer that there is a similarity, figure 5 .

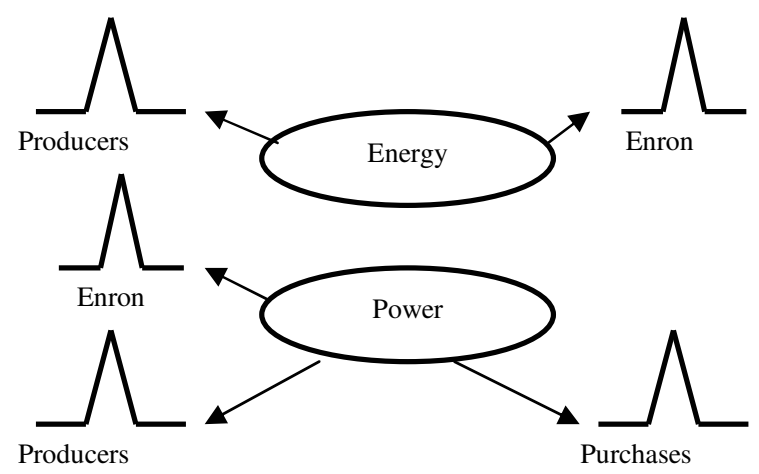

FIGURE 5: Similarity using Strength of association

\footnotetext{
${ }^{3}$ Dasovich Email account consists of 21,433,538 tokens, 93,097 types, the Nucleate "energy" occurs 62,153 times, and the +/-5 token width vocabulary is 16821.
} 
Levy \& Bullinaria, use vectors based on collocate frequencies; we prefer a strength of association measure based on how unusually frequent and how tightly the distance relationship is constrained, hence $Q w$. We derive the formula 4 to compare similarities:

$$
S=\frac{2 \sum Q \mathcal{W}(a)_{i} \times Q \mathcal{W}(b)_{i}}{\sum Q \mathcal{W}(a)_{i}^{2}+\sum Q w(b)_{i}^{2}}
$$

$S=$ Similarity where $1=$ Identical

$i=$ matching collocate

$$
\begin{aligned}
& Q W(a)_{i}=Q w \text { of collocate } i \text { of nucleate } a \\
& Q_{(b)_{i}}=Q w \text { of collocate } i \text { of nucleate } b
\end{aligned}
$$

When the comparison is made between identical corpora, the weirdness measures will cancel out. This allows us to compare variations in term use across different subsamples i.e. examine subsample variations.

\subsection{Jeff Dasovich's energy, power \& contracts}

We initially removed all Dasovich3 energy collocations of frequency less than 11 , since our confidence in the peak analysis results across 10 collocation positions would be low. This left 3992 collocations to analyse.

We ranked the collocations of energy the formulas for $Q$ _peak, Weirdness and Qw, using a collocation window size of $+/-5$, and then compared the rankings against the BNC top 2000 stop list. Figure 6 below shows how these matched BNC stop list words are distributed (as a \% accumulation) within these different rankings.

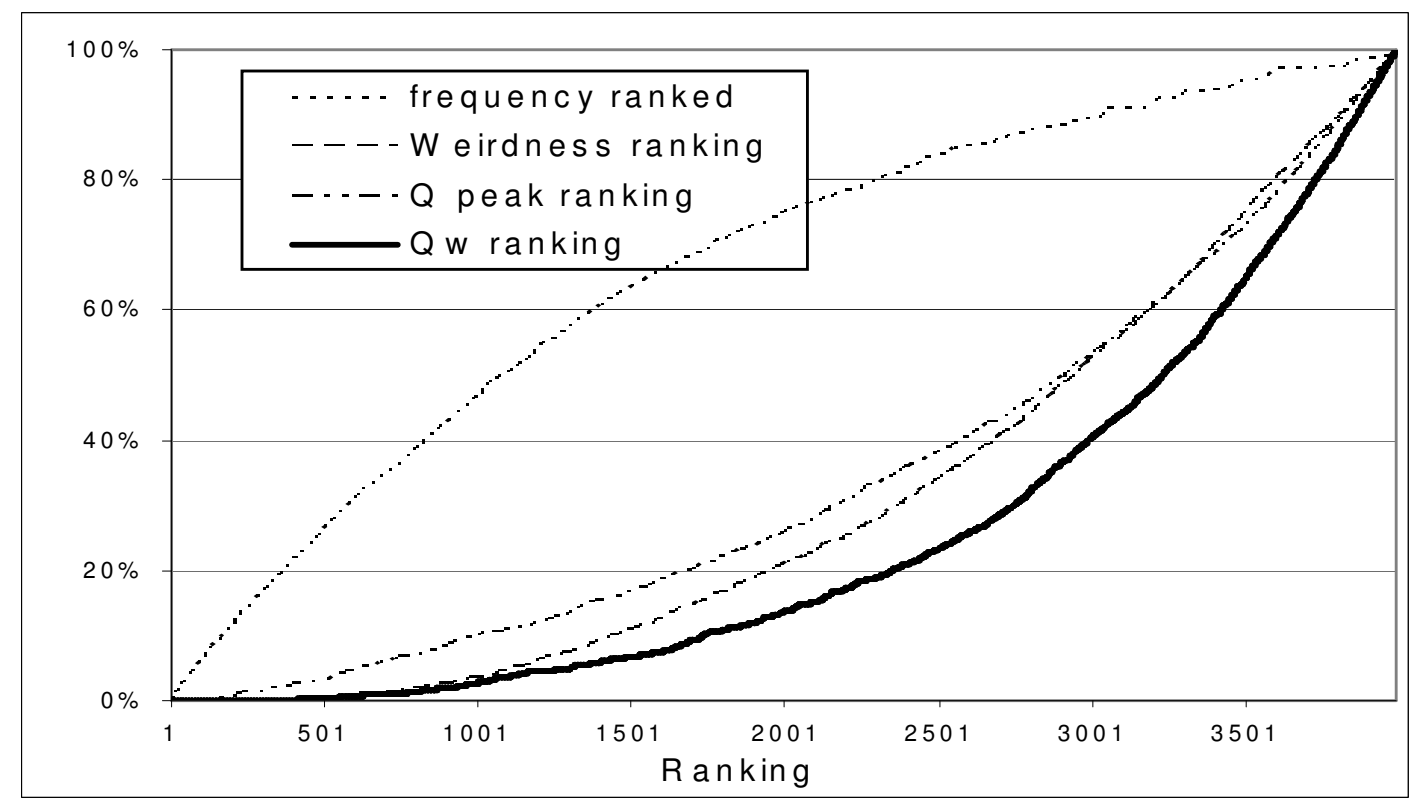

FIGURE 6: BNC top 2000 distribution comparisons

The BNC words have been pushed to the right by each ranking method, without ending up simply inverse frequency correlated. Using spearman' coefficient, the correlation of $Q w$ ranking and Frequency ranking is 0.30861 , indicating little correlation, which is good.

Table 7 shows the top 10 and bottom 10 ranking words for each ranking method. BNC top 2000 words appear in bold.

Next we choose a cut off point for Qw and exclude all collocations with a Qw less than 1.2 (3606 words in total a $90 \%$ reduction). The frequency ranked table 8 shows the top 20 of the remaining 386 automatically selected collocations. Again the BNC top 2000 words appear in bold, these words would have been removed if these 2000 words had been used as a conventional stop list.

From this we can surmise Dasovich's main interests/activities/correspondence in relation to "energy". Similarly we can extract for Dasovich's use of "Power" ${ }^{4}$ the top and bottom rankings (Table 9 ) and after auto stop word removal produce a top 20 ranking (Table 10) and similarly for "Contracts" (Table 11).

\footnotetext{
${ }^{4}$ The nucleate "power" occurs 82,252 times, and the $+/-5$ token width vocabulary is 17,084 .
} 


\begin{tabular}{lllll}
\hline Rank & Frequency & Weirdness & Q peak & Qw \\
\hline 1 & the & dasovich & webmaster & inbox \\
2 & to & jdasovic & castano & sempra \\
3 & of & http & oom & dasovich \\
4 & and & enron & fuelcell & jdasovic \\
5 & in & ferc & rfb & pdt \\
6 & a & calpine & blvd & haas \\
7 & for & cpuc & dasovitch & iep \\
8 & on & dynegy & eme & greenmountain \\
9 & crisis & venturewire & federa & ferc \\
10 & that & inbox & barretto & caem \\
$\sim$ & $\sim$ & $\sim$ & $\sim$ & $\sim$ \\
3983 & corner & towards & more & when \\
3984 & filled & stood & when & got \\
3985 & branch & man & southern & made \\
3986 & popular & looked & fuel & er \\
3987 & broken & er & calpine & she \\
3988 & sitting & her & davis & did \\
3989 & dismissal & britain & this & his \\
3990 & accompanied & shook & made & him \\
3991 & movement & men & utilities & shook \\
3992 & stood & aye & as & her \\
& TABLE 7: Dasovich "Energy" Top and bottom ranking comparisons
\end{tabular}

\begin{tabular}{llllll}
\hline Rank & Word & Freq & Rank & Word & Freq \\
\hline 1 & crisis & 5053 & 11 & dow & 1446 \\
2 & california & 4374 & 12 & trading & 1254 \\
3 & commission & 4177 & 13 & producers & 1198 \\
4 & federal & 3595 & 14 & renewable & 1141 \\
5 & enron & 3399 & 15 & costs & 1118 \\
6 & regulatory & 2996 & 16 & markets & 1095 \\
7 & companies & 2368 & 17 & jones & 1064 \\
8 & duke & 2042 & 18 & sempra & 1014 \\
9 & prices & 1825 & 19 & wholesale & 1004 \\
10 & reliant & 1638 & 20 & senate & 778
\end{tabular}

TABLE 8: Top 20 Dasovich "Energy" collocations after Auto Stop word removal.

\begin{tabular}{lllll}
\hline Rank & Frequency & Weirdness & Q peak & Qw \\
\hline 1 & the & dasovich & conf & charset \\
2 & to & charset & plain & inbox \\
3 & of & http & abag & hartsoe \\
4 & and & enron & privileged & steffes \\
5 & in & ferc & capitalists & etringer \\
6 & for & calpine & hartsoe & newenergy \\
7 & a & cpuc & thefts & dabhol \\
8 & that & dynegy & orion & abag \\
9 & on & steffes & amassing & dasovich \\
10 & the & inbox & nth & cpuc \\
$\sim$ & $\sim$ & $\sim$ & $\sim$ & $\sim$ \\
4278 & bray & influences & a & sometimes \\
4279 & assn & stolen & the & when \\
4280 & dlrs & towards & as & got \\
4281 & skeptical & hundred & that & programme \\
4282 & sw & looked & over & his \\
4283 & summ & er & on & then \\
4284 & eix & her & between & er \\
4285 & multiyear & church & back & her \\
4286 & transwestern & eyes & because & war \\
4287 & unsubscribe & programme & when & she \\
\hline
\end{tabular}

TABLE 9: Dasovich "Power" Top and bottom ranking comparisons 


\begin{tabular}{llllll}
\hline Rank & Word & Freq & Rank & Word & Freq \\
\hline 1 & plants & 6132 & 11 & producers & 1019 \\
2 & plant & 3918 & 12 & dwr & 991 \\
3 & generators & 2549 & 13 & angeles & 722 \\
4 & dabhol & 2498 & 14 & megawatts & 712 \\
5 & wholesale & 2464 & 15 & dpc & 705 \\
6 & enron & 2209 & 16 & megawatt & 665 \\
7 & grid & 1772 & 17 & ferc & 662 \\
8 & electric & 1591 & 18 & blackouts & 569 \\
9 & purchases & 1384 & 19 & marketers & 485 \\
10 & co & 110 & 20 & sellers & 471
\end{tabular}

TABLE 10: Top 20 Dasovich "Power" collocations after Auto Stop word removal.

\begin{tabular}{llllll}
\hline Rank & word & Freq & Rank & word & Freq \\
\hline 1 & expiring & 1133 & 11 & stocks & 1049 \\
2 & schedules & 1130 & 12 & sues & 1042 \\
3 & nd & 1125 & 13 & analyze & 1041 \\
4 & beforce & 1123 & 14 & billions & 1039 \\
5 & retroactive & 1122 & 15 & finalize & 1035 \\
6 & multiyear & 1121 & 16 & steven & 1034 \\
7 & sacramento & 1120 & 17 & gen & 1031 \\
8 & angelides & 1119 & 18 & byline & 1030 \\
9 & sempra & 1118 & 19 & favorable & 1029 \\
10 & outs & 1052 & 20 & fw & 1028
\end{tabular}

TABLE 11: Top 20 Dasovich "Contracts" collocations after Auto Stop word removal

Measuring similarity of "energy" and "power" we might expect some degree of similarity of context, knowing a priori that Enron is an Energy/power company. Using formula 4 for "energy" and "power" for all tokens that match get a similarity measure of 0.87 , indicating a close similarity. As a comparison the similarity between "power" and "contracts" 6 is 0.26 , indicating a more distant relationship.

To complete the analysis we would need to determine whether the relationship is synonymous or hierarchical and here we may need to consider patterns of exclusions as identified above.

\subsection{Much-more validation}

To validate the approach we have performed the same operations using the Muchmore corpus ${ }^{7}$ on "case" 8 , "study" and "blood" ${ }^{10}$ and the results are presented in table 12. Here the Qw stop word cut off s set to 1.0. Again the BNC top 2000 words appear in bold, and we can again infer the dominant semantic associations.

\begin{tabular}{lllllll}
\hline Rank & $\begin{array}{l}\text { patients } \\
\text { word }\end{array}$ & freq & $\begin{array}{l}\text { Study } \\
\text { Word }\end{array}$ & $\begin{array}{l}\text { freq } \\
\text { woord }\end{array}$ & freq \\
\hline 1 & underwent & 154 & Aim & 320 & pressure & 237 \\
2 & chronic & 140 & present & $\mathbf{2 3 9}$ & flow & 176 \\
3 & suffering & 128 & prospective & 209 & loss & 75 \\
4 & postoperative & 116 & retrospective & 116 & arterial & 60 \\
5 & undergoing & 105 & evaluate & 107 & cerebral & 49 \\
6 & trauma & 99 & performed & 56 & vessels & 42 \\
7 & tumor & 95 & randomized & 50 & gas & 41 \\
8 & coronary & 89 & multicenter & 33 & samples & 37 \\
9 & fractures & 88 & efficacy & 22 & peripheral & 35 \\
10 & tumors & 79 & Shows & $\mathbf{2 0}$ & autologous & 29 \\
11 & metastases & 69 & analyze & 19 & systolic & 27 \\
12 & consecutive & 68 & Pilot & 17 & venous & 23 \\
13 & artery & 66 & diagnostic & 15 & transfusions & 23 \\
14 & atrial & 65 & analyzed & 15 & gases & 17 \\
15 & prognosis & 62 & therapy & 15 & intraoperative & 15 \\
16 & carcinoma & 59 & fractures & 14 & diastolic & 14 \\
17 & resection & 56 & complications & 13 & uterine & 13 \\
18 & malignant & 52 & longitudinal & 12 & glucose & 13 \\
19 & critically & 51 & investigates & 12 & patch & 12 \\
20 & according & 51 & demonstrates & 12 & cardiac & 12
\end{tabular}

TABLE 12: MuchMore Top 20 "patients", "study" \& "blood" collocations: after auto stop word removal.

\footnotetext{
${ }^{5}$ Stop words are not removed, their influence is naturally deprecated by small values for Q_peak and weirdness.

${ }^{6}$ The nucleate "contracts" occurs 11,492 times, and the +/-5 token width vocabulary is 5,185.

${ }^{7}$ The Springer Muchmore corpus of 7823 medical abstracts, vocabulary is 35033 types, total size is 1081124 tokens.

8 "patients" occurs 9,410 times, ranked 10 by frequency with a weirdness of 50

9 "study" occurs 1,979 times, ranked 40 by frequency with a weirdness of 8.3

10 "blood" occurs 1,097 times, ranked 87 by frequency with a weirdness of 10
} 
Using equation 4, for all matching words, similarity calculations indicate "patients" similarity to "study" (0.87) is high and "patients" similarity to "blood" $(0.38)$ is low, however for "case" and "cases" "11 the similarity is 0.94 indicating more similarity as we would expect.

\section{DISTRIBUTIONS OF DISTRIBUTIONS}

We consider, finally, whether such results provide a set of predictable patterns, hence predictable keywords and collocations, and stop lists that can be used accurately with smaller proportions of the same corpus. In particular, we consider:

- Verticals: splitting the Enron corpus into sub-corpora, e.g. by user names - 150 separate users. Analysing and comparing use of language in these sub-corpora, with initial focus on "confidential" to demonstrate initial discriminance.

- Horizontals: marking out boundaries within texts (emails). A software component has been created to facilitate the identification and marking and collocation analysis is then used to provide the statistical evidence to reveal and investigate these less dominant contexts.

- Cumulative: For smaller collections we propose using the cumulative shape information of the collocates to infer entropy on the nucleate.

- $\quad$ Aggregate: To produce an aggregate stop list for general use, we propose to initially choose a nucleate at random and then to choose the next nucleate based on the collocation rankings and corpus collocation coverage. Once the net coverage overlap is greater than the unprocessed area, combine the nucleate collocation stop lists to build an aggregate stop list for the whole corpus.

These four orientations will assist both to identify other lower frequency distributions and to enable the accurate classification of body text versus banner.

Figure 7, below, demonstrates our rationale for verticals: we split collocation analysis across 4 quarters and investigated dominant patterns for the collocate "privileged" with "confidential". One quarter appeared to produce unusual behaviour in contrast to the relatively similar distributions for the remaining quarters, evident in the difference in heights of the third bar at collocate positions L2 and R2. Either we were detecting different types of banners, or there was some other significant explanation such as legal discussions - for this.

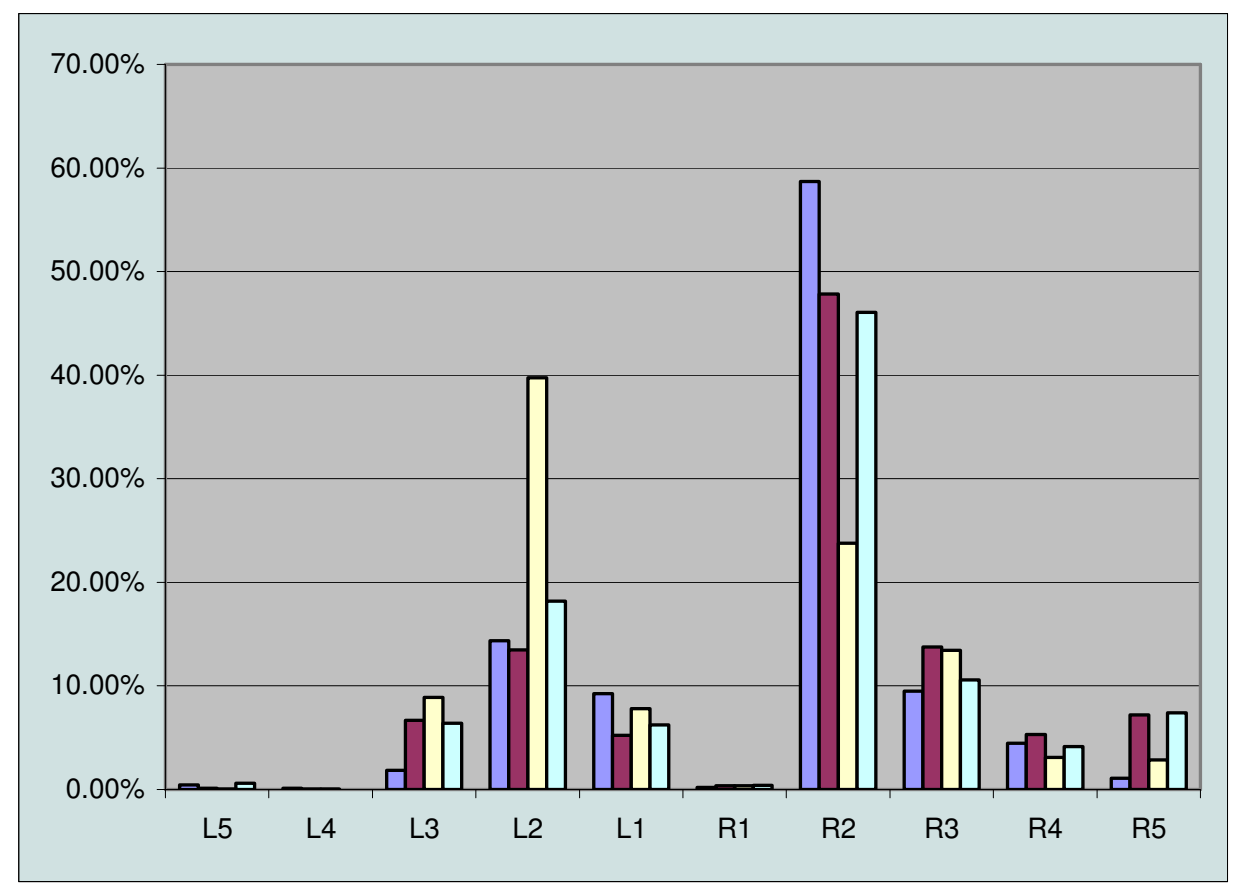

FIGURE 7: Vertical Spitting

\section{CONCLUSIONS}

Our aim is to be able to take an arbitrary text collection and automatically apply necessary and appropriate data cleansing techniques to it, have it produce its own stop word list, and provide a more robust ground for corpus analysis. This is necessary not only for email corpora such as that of Enron, but also for analysis of Blogs, Wikis, Web- Corpora, and other types of social or specialist collections.

Our approach has been evaluated and validated using publically available corpora, and tested against a BNC frequency list based stop list.

We have demonstrated that we can identify to some extent, certain kinds of information pollution within such corpora, and use statistical approaches to distributions for identifying stop words and lexical semantics. The approach attempts to remove assumptions relating to the text under analysis; it uses the collocation patterns associated with stop words combined with the relative frequency properties of stop words to derive the stop word list. We have demonstrated the ability to measure distributional lexical similarity and have compared "power" with 
"energy" and "contracts", showing that "power" is closely related to "energy" and only distantly related to "contracts". However further work is required to bench mark the metric and understand how similarity is scaled in the range 0 to 1 , and also in due course to extend and benchmark the semantic representation approach in this paper using the TOEFL® Test $^{12}$ in a similar manner to Bullinaria \& Levy (2006).

\section{BIBLIOGRAPHY}

[1] Bekkerman R; A. McCallum. \& G. Huang, 2004, "Automatic Categorization of Email into Folders: Benchmark Experiments on Enron and SRI Corpora". (Massachusetts).

[2] Blei D M; A. Y. Ng; M. I. Jordan, 2003, Latent Dirichlet Allocation, Journal of Machine Learning Research.

[3] Budanitsky A.; G. Hirst, 2006, Evaluating WordNet-based Measures of Lexical Semantic Relatedness, Association for Computational Linguistics, MIT Press.

[4] Bullinaria J.A; J.P. Levy, 2006, Extracting Semantic Representations from Word Co-occurrence Statistics: A Computational Study, Behavior Research Methods.

[5] Cooke N; L. Gillam; A. Kondoz, 2007a, Best Kept Secrets of Corpus Linguistics, L2007 Birmingham.

[6] Cooke N.; L. Gillam; A. Kondoz, 2007b, IP protection: Detecting Email based breaches of confidence, IAS2007 Manchester .

[7] Fumera G; I. Pillai; F. Roli, 2006, Spam Filtering Based On The Analysis Of Text Information Embedded Into Images, Machine Learning Research 6: 2699-2720,

[8] Gillam L.; K. Ahmad, 2005, Pattern mining across domain-specific text collections. LNAI 3587, pp 570-579.

[9] Gillam L.; M. Tariq; K Ahmad, 2005, Terminology and the Construction of Ontology, Terminology 11(1), pp55-

81. John Benjamins Publishing Company, ISSN 0929-9971; E-ISSN 1569-9994.

[10] Hiemstra D.; F. de Jong, 2001, Statistical Language Models and Information Retrieval: natural language processing really meets retrieval, Glot International 5(8), pages 288-294, http://www.glotinternational.com.

[11] Jabbari S.; B. Allison; D. Guthrie; L. Guthrie, 2006, Towards the Orwellian Nightmare Separation of Business and Personal Emails, Proceedings of the COLING/ACL 2006 Main Conference Poster Sessions, pages 407-41.

[12] Klimt B.; Y. Yang, 2004, The Enron Corpus: A New Dataset for Email Classification Research, ECML 2004: 217-226 Language Technologies Institute, Carnegie Mellon University.

[13] Levy J.P.; J.A. Bullinaria, 2001, Learning Lexical Properties from Word Usage Patterns: Which Context Words Should be Used?

[14] Li, W. (1992) "Random texts exhibit Zipf's-law-like word frequency distribution". IEEE Transactions on Information Theory 38(6) pp1842-1845.

[15] Lo. R.T; B. He; I. Ounis, 2005, Automatically building a stopword list for an information retrieval system, The Journal on Digital Information Management: special issue on the 5th Dutch-Belgian Information Retrieval Workshop (DIR 2005)

[16] Manning, C. and Schütze, H. (1999) "Foundations of Statistical Natural Language Processing". MIT Press. Cambridge, MA.

[17] Pustejovsky, J.; P. Anick; and S. Bergler, (1993), "Lexical Semantic Techniques for Corpus Analysis", Computational Linguistics 19(2): 331-358

[18] Sinka M. P; D.W. Corne, 2003, Evolving Better Stoplists for Document Clustering and Web Intelligence Design and application of hybrid intelligent systems, 1015-1023.

[19] Smadja F, 1993, Retrieving collocations from text: Xtract, Computational Linguistics, 19(1) pp143-178. Oxford University Press.

[20] Trumbach C.C.; D. Payne, 2007, Identifying synonymous concepts in preparation for technology mining, Journal of Information Science 2007; 33; 660 originally published online Jun 14.

[21] Watts R.J.; A.L. Porter; D. Zhu, 2002, Factor Analysis Optimization: Applied on Natural Language Knowledge Discovery, Committee on Data for Science and Technology 2002: Frontiers of Scientific and Technical Data: Proceedings of the 18th International Conference CODATA 2002

[22] Wilbur W. J.; K. Sirotkin, 1992, The automatic identification of stop words, Journal of Information Science18; 45

[23] Yavuz, D. (1974) "Zipf's law and entropy" (Corresp.) IEEE Transactions on Information Theory, 20 (5). p650

[24] Zipf, G.K. (1949) "Human Behavior and the Principle of Least Effort". Hafner, New York.

\section{AUTHOR BIOGRAPHIES}

Neil Cooke BSc Computer \& Control Systems Lanchester Polytechnic Coventry UK 1981 (now University of Coventry), Chartered Engineer 1987, Fellow of the Institute of Engineering Technology 2005. Studying part-time for a PhD, at the University of Surrey. The author has 18 years of experience in the field of information assurance engineering for the UK government's National Technical Authority for Information Assurance. Prior to this the author was an avionics and marine systems engineer.

Lee Gillam PhD in Artificial Intelligence (Surrey, 2004); BSc in Mathematics and Computer Science (Surrey, 1995). Member of the British Computer Society (MBCS) and Chartered IT Professional (CITP). Currently a Lecturer in the Department of Computing at the University of Surrey. Previous publications and research in the areas of Ontology Learning, Metadata and Grid Computing Systems.

${ }^{12}$ http://www.ets.org/portal/site/ets/ 